\title{
Conjoining auditory and visual features during high-rate serial presentation: Processing and conjoining two features can be faster than processing one
}

\author{
DAVID L. WOODS \\ University of California, Davis, California \\ CLAUDE ALAIN \\ University of California, Davis, Califormia \\ Rotman Research Institute of the Baycrest Center, Toronto, Ontario, Canada \\ and University of Toronto, North York, Ontario, Canada \\ and \\ KEITH H. OGAWA \\ University of Califormia, Davis, Califormia \\ and St. Mary's College, Moraga, California
}

\begin{abstract}
The time required to conjoin stimulus features in high-rate serial presentation tasks was estimated in auditory and visual modalities. In the visual experiment, targets were defined by color, orientation, or the conjunction of color and orientation features. Responses were fastest in color conditions, intermediate in orientation conditions, and slowest in conjunction conditions. Estimates of feature conjunction time (FCT) were derived on the basis of a model in which features were processed in parallel and then conjoined, permitting FCTs to be estimated from the difference in reaction times between conjunction and the slowest single-feature condition. Visual FCTs averaged $17 \mathrm{msec}$, but were negative for certain stimuli and subjects. In the auditory experiment, targets were defined by frequency, location, or the conjunction of frequency and location features. Responses were fastest in frequency conditions, but were faster in conjunction than in location conditions, yielding negative FCTs. The results from both experiments suggest that the processing of stimulus features occurs interactively during early stages of feature conjunction.
\end{abstract}

Much of what is known about the conjoining of features is based on visual search experiments in which subjects search for target stimuli in arrays of distractors. In such experiments, the time required to detect a target increases with the number of distractors. When targets are defined by simple features, such as color, search slopes relating reaction time (RT) to set size are typically shallow (see, e.g., Treisman \& Gelade, 1980). However, when targets are defined by a conjunction of features, slopes are steeper (Treisman \& Sato, 1990; Wolfe, 1994). A parallel/serial $(\mathrm{P} / \mathrm{S})$ model in which stimulus features are processed independently and in parallel followed by a capacity-limited stage of feature conjunction (Treisman, 1988) can account for these results.

The research was supported by grants from the NINDS (NS-32893) and the VA Research Service to D.L.W. and by an FRSQ postdoctoral fellowship to C.A. Special thanks to Dell Rhodes, Steve Thomas, and Bill Yund for careful readings of earlier versions of this manuscript. Correspondence should be addressed to D. L. Woods, University of California, Davis, Neurology Service (612/127), Northern California System of Clinics, 150 Muir Road, Martinez, CA 94553 (e-mail: dlwoods@ ucdavis.edu).
The steeper slopes in conjunction search include contributions from the time required to spatially displace attention over the stimulus array (Treisman \& Gelade, 1980). Spatial precuing speeds target detection so that precued targets in large arrays can be processed as rapidly as targets in smaller arrays (Klein \& Farrell, 1989; Prinzmetal, Presti, \& Posner, 1986). However, in serial models, the time required to spatially displace attention includes the time required to process and conjoin the features of stimuli at the focus of attention. Attentional displacement also includes contributions from other processing operations. For example, when subjects search the display they preattentively group individual stimuli in order to direct attention to stimulus objects (Driver, Baylis, \& Rafal, 1992); displace attention to objects sharing target features; analyze those objects; and disengage, move, and reengage attention on other objects in the display (Posner \& Driver, 1992). Thus, set-size functions in visual search would be determined jointly by the time required to displace attention (including the time to engage and disengage attention) and the time required to process and conjoin features at the attentional focus. 


\section{Rapid Serial Visual Presentation}

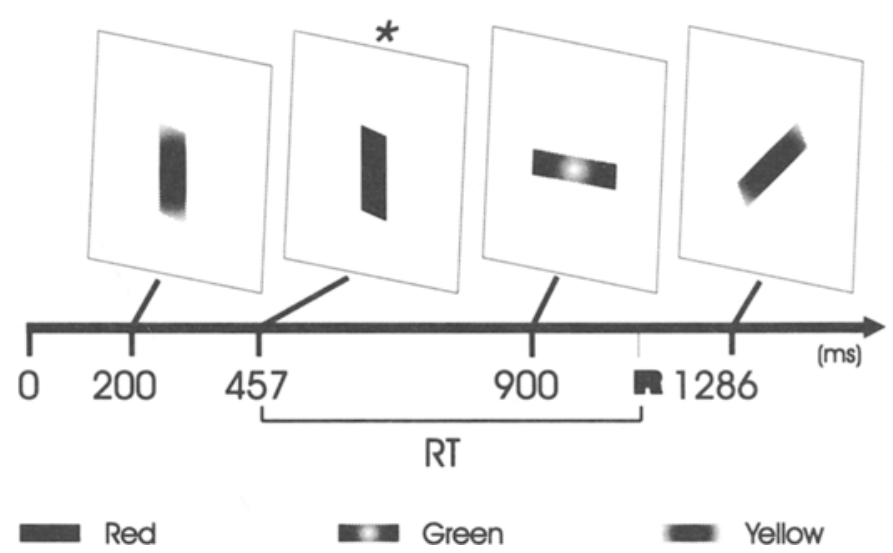

Figure 1. Subjects viewed sequences of equiluminant bars varying randomly in color (red, green, yellow) and orientation (vertical, diagonal, horizontal) and presented against an equiluminant, continuously present gray background. In color conditions, subjects responded to targets of a designated color, regardless of orientation. In orientation conditions, they responded to targets of a designated orientation, regardless of color. In conjunction conditions, they responded to targets with a prespecified color and orientation. A segment of the conjunction sequence, "red vertical," is shown. $R$, response; *target; $R T$, reaction time.

In the present study, we analyzed feature processing and conjunction with foveally presented stimuli in a rapid serial presentation (RSP) task. Since the stimuli were presented at a fixed location, an estimate of feature conjunction time (FCT) could be derived that was uninfluenced by the spatial displacement of attention. The estimate of FCT was based on a $\mathrm{P} / \mathrm{S}$ model postulating parallel feature processing followed by a serial stage of feature conjunction. Thus, FCTs were computed by subtracting RTs in the most difficult single-feature condition from RTs in featureconjunction conditions.

\section{EXPERIMENT 1}

In Experiment 1, FCTs were analyzed for colored bars presented at fixation. We anticipated that FCTs would be smaller than the difference in search slopes between featureconjunction and single-feature conditions (Treisman \& Gelade, 1980) because search slopes include contributions from processing operations (stimulus grouping, attentional displacement, disengagement, etc.) eliminated in an RSP paradigm with stimuli presented at fixation.

\section{Method}

Subjects. Twelve volunteers ( 4 men and 8 women; age range, 18-45 years) participated. All subjects were right-handed with normal or corrected-to-normal visual acuity ( $20 / 20$ by self-report).

Stimuli and Tasks. Nine different colored bars were made by combining three colors (red, green, or yellow) and three orientations (vertical, horizontal, or right diagonal at a $45^{\circ}$ angle from the lower left to the upper right). The luminance of the bars was psychophysically equated with each other and with the gray background using heterochromatic photometry. Actual luminance values were red $4.14 \mathrm{~cd} / \mathrm{m}^{2}$ (CIE coordinates $\left.x=0.616, y=0.357\right)$, green $4.98 \mathrm{~cd} / \mathrm{m}^{2}$ (CIE coordinates $x=0.294, y=0.611)$, and yellow $4.83 \mathrm{~cd} / \mathrm{m}^{2}(\mathrm{CIE}$ coordinates $x=0.463, y=0.471)$, gray background $\left(5.13 \mathrm{~cd} / \mathrm{m}^{2}, \mathrm{CIE}\right.$ coordinates $x=0.285, y=0.322$ ). The bars subtended $1.08 \times 0.29^{\circ}$ in visual angle and were presented at central fixation on a monitor subtending $14.2^{\circ}$ wide and $10.6^{\circ}$ high at a distance of $160 \mathrm{~cm}$ from the subject. Stimulus duration was $57.1 \mathrm{msec}$ (four video frames). Stimulus onset asynchronies (SOAs) ranged from 171 to $457 \mathrm{msec}$ (mean, $314 \mathrm{msec}$ ) according to a rectangular distribution.

The stimuli were presented in three different conditions: color, orientation, and conjunction (Figure 1). There were three repetitions of each color and orientation condition. In each, the subjects pressed a button as rapidly as possible to the occurrence of the target feature regardless of other features of the stimulus. For example, during the attend-red condition, subjects responded to red bars regardless of orientation. In conjunction conditions, subjects responded to a designated conjunction of color and orientation. For example, in the red-vertical condition, subjects responded only to red vertical bars.

In all conditions, targets constituted one ninth of all stimuli. In conjunction conditions, this probability was derived from the random combination of color and orientation $(1 / 3 * 1 / 3)$. In color and orientation conditions, the probability of the target feature was reduced to $11.1 \%$ with the two other features each presented on $44.4 \%$ of the trials. Targets were presented at mean intervals of $3.34 \mathrm{sec}$ (range, $0.3-15 \mathrm{sec}$ )

Twenty-seven blocks (nine color, nine orientation, and nine conjunction conditions) were presented during the experiment. Each 3.67-min block contained 504 nontargets and 63 targets in a single randomized sequence. The order of block presentation was randomized within and between subjects. In all conditions, subjects were encouraged to be both fast and accurate in their responses.

Data analysis. Correct responses were defined as buttonpresses occurring between 200 and $1,000 \mathrm{msec}$ after target onset. Responses not preceded by a target within $1,000 \mathrm{msec}$ were classified as false alarms (FAs). The FA rate was expressed as a percentage of the num- 
Table 1

Mean Percentage of Misses and False Alarms

and Reaction Times (RTs, in Milliseconds)

for the Different Conditions of Experiment 1, Averaged Over Stimuli of Different Color and Orientation

\begin{tabular}{lccc}
\hline Condition & Misses (\%) & False Alarms (\%) & Mean RT \\
\hline Conjunction & 0.8 & 1.1 & 494 \\
Orientation & 0.9 & 0.6 & 477 \\
Color & 0.8 & 0.4 & 410 \\
\hline
\end{tabular}

ber of nontarget stimuli in a block. Mean RTs, FA rates, and miss rates were compared in separate analyses of variance (ANOVAs) with stimulus color, stimulus orientation, and attention condition as factors.

The pattern of stimuli that preceded FAs was examined in detail in the conjunction conditions. FAs were attributed to the first stimulus occurring in a 300 - to $800-\mathrm{msec}$ window preceding the FA buttonpress. The analysis window for FA-eliciting stimuli was based on the assumptions that FA RTs would be similar to RTs to correctly detected targets, with the additional constraint that the FA window be narrow enough to include only one or two stimuli. The miscombination of features across stimuli was further examined by evaluating the extent to which FAs followed pairs of stimuli with repeated or complementary target features.

\section{Results}

Overview. Mean RTs averaged $460 \mathrm{msec}$ (range across subjects, 401-515 msec). Subjects were highly accurate: Misses occurred on $0.8 \%$ of target presentations. The mean FA rate was $0.7 \%$.

Performance measures from color, orientation, and conjunction conditions are presented in Table 1 . Subjects responded faster in color conditions $(410 \mathrm{msec})$ than in orientation conditions $(477 \mathrm{msec})$ or conjunction conditions (494 $\mathrm{msec})$. This produced a highly significant condition main effect $[F(2,22)=57.1, p<.001]$. A pairwise comparison showed that subjects were faster in color than in orientation conditions $[F(1,11)=35.2, p<.001]$. The difference in performance in color and orientation conditions was large and there was no overlap in mean RTs between the different single-feature subconditions (RT range, $366-436 \mathrm{msec}$ in color conditions vs. $455-500 \mathrm{msec}$ in orientation conditions). Eleven of the 12 subjects showed faster responses in color than in orientation conditions (RT difference $=41-134 \mathrm{msec}$ ), whereas the 12 th subject showed similar RTs in the two conditions ( $413 \mathrm{vs.} 415 \mathrm{msec}$ ). Mean RTs in both color and orientation conditions were faster than those in conjunction conditions $[F(1,11)=$ $210.2, p<.001$, and $F(1,11)=6.4, p<.05$, respectively].

There were no significant differences in miss rate among conditions $[F(2,22)=0.37$; see Table 1]. However, FA rates varied with condition $[F(2,22)=14.47, p<.001]$, being higher in conjunction than in orientation conditions $[F(1,11)=14.6, p<.001]$ and higher in orientation than color conditions $[F(1,11)=6.3, p<.03]$.

Anisotropies in the processing of target features. Performance measures associated with the processing of targets of different color are presented in Table 2. Red stimuli were detected more rapidly than yellow or green stimuli $[F(2,22)=34.6, p<.001]$. Pairwise comparisons showed that subjects were faster to respond to red than to green or yellow stimuli. Faster responses to red stimuli were not due to a speed-accuracy tradeoff: Miss rates were similar for the different colors, and FA rates were lower for red than for green or yellow stimuli $[F(1,11)=6.3, p<.03]$.

Performance measures associated with the processing of targets of different orientation are presented in Table 3. In orientation conditions, vertical targets were detected more rapidly than diagonal or horizontal targets $[F(2,22)=$ $6.2, p<.01]$. However, unlike the color anisotropiesseen in all conditions-no significant orientation effects were found in color or conjunction conditions. This resulted in a significant condition $\times$ orientation interaction $[F(4,44)=4.38, p<.01]$

Feature conjunction. Table 4 shows the mean RTs for each of the nine different stimuli in color, orientation, and conjunction conditions. Also shown are estimates of FCTs, derived for each stimulus by subtracting the mean RT in the slowest single-feature condition (always orientation) from the RT to the same stimulus in conjunction conditions. For example, FCTs for red-vertical stimuli were derived by subtracting RTs to red-vertical stimuli in attend-vertical conditions from RTs to the same stimuli in attend-redvertical conditions. Mean FCTs averaged $17 \mathrm{msec}$.

FCTs varied for different stimuli, producing a color $X$ orientation interaction $[F(4,44)=4.45, p<.02]$. Small positive FCTs were obtained for most stimuli (range, 4-38 $\mathrm{msec}$ ), but negative FCTs were seen for yellowhorizontal bars whereas yellow-diagonal bars had an FCT of zero.

We also found that FCTs were negative for some subjects. Ten subjects showed positive mean FCTs (range, 1-60 msec), but 2 subjects showed negative mean FCTs $(-26$ and $-9 \mathrm{msec}$ ). For the latter subjects, RTs were faster in conjunction than in orientation conditions. Data from the individual conditions from individual subjects showed that responses were universally faster in color than in conjunction conditions. However, this was not true for conjunction/ orientation comparisons: Responses in conjunction conditions were faster on $27 \%$ of the individual comparisons.

Since a P/S model of feature processing assumes that feature conjunction must await the termination of singlefeature processing stages, the occurrence of negative FCTs calls into question the $\mathrm{P} / \mathrm{S}$ model. The $\mathrm{P} / \mathrm{S}$ model also makes predictions about response variance: RT distributions in conjunction conditions should show increased means in comparison with single-feature conditions, but reduced variances. Variances should be reduced because the RT distribution in conjunction conditions would depend on the slower of the two single-feature

Table 2

Mean Reaction Times (in Milliseconds)

for Targets of Different Colors in Color, Orientation, and Conjunction Conditions

\begin{tabular}{cccc}
\hline & \multicolumn{3}{c}{ Condition } \\
\cline { 2 - 4 } Target Color & Color & Orientation & Conjunction \\
\hline Red & 366 & 426 & 444 \\
Yellow & 427 & 502 & 508 \\
Green & 436 & 502 & 529 \\
\hline
\end{tabular}


Table 3

Mean Reaction Times (in Milliseconds)

for Targets of Different Orientations in

Color, Orientation, and Conjunction Conditions

\begin{tabular}{lccc}
\hline \multirow{2}{*}{$\begin{array}{c}\text { Target } \\
\text { Orientation }\end{array}$} & Color & Orientation & Conjunction \\
\cline { 2 - 4 } Vertical & 412 & 455 & 488 \\
Diagonal & 413 & 475 & 491 \\
Horizontal & 406 & 500 & 503 \\
\hline
\end{tabular}

processes. Very fast RTs in conjunction conditions, associated with rapid orientation processing, would not occur because on those trials, color, not orientation, would become the rate-limiting feature. Thus, in comparison with orientation conditions, conjunction conditions would show increased mean RTs but reduced RT variances.

We therefore performed an analysis of within-subjects RT variances. Significant main effects were seen for condition [mean standard deviation of within-subjects RTs: color $=114 \mathrm{msec}$, orientation $=139 \mathrm{msec}$, conjunction = $160 \mathrm{msec}, F(2,22)=24.9, p<.001]$. All pairwise comparisons reached significance [range, $F(1,11)=9.2$ to 46.4 , $p<.01$, for different comparisons]. Contrary to the predictions of the P/S model, variance increased in conjunction in comparison with orientation conditions. Moreover, RT variances in conjunction conditions were larger than the mean FCT. This implies that negative FCTs would have frequently obtained on individual trials. Again, the small magnitude of FCT poses problems for the P/S model.

In fact, even the small FCT obtained was likely inflated by its method of calculation. Despite the fact that there was no overlap in mean RTs in color and orientation conditions, variance analysis implies that on some trials, color processing would have finished after orientation processing. For example, assuming a normal distribution of color (mean RT $=410 \mathrm{msec}, S D=114 \mathrm{msec}$ ) and orientation (mean RT $=477 \mathrm{msec}, S D=139 \mathrm{msec}$ ) processing, color processing would be expected to finish after orientation processing on $20 \%-35 \%$ of the trials in conjunction conditions. Since the estimate of FCT was based on the assumption that orientation processing always finished after color processing, this would have artifactually inflated FCTs.

False alarms. In conjunction conditions, one stimulus was the target and eight different stimuli were distractors. Two distractors shared target color $(\mathrm{C}+\mathrm{O}-)$ and two shared target orientation $(\mathrm{C}-\mathrm{O}+)$. The remaining four distractors shared neither target feature $(\mathrm{C}-\mathrm{O}-)$. Not surprisingly, FAs occurred more frequently following stimuli with target features than following $\mathrm{C}-\mathrm{O}-$ distractors $[1.87 \% \mathrm{vs}$. $0.24 \%, F(1,11)=29.8, p<.001]$. C+O- and $\mathrm{C}-\mathrm{O}+$ distractors produced similar percentages of FAs $(1.84 \%$ vs. $1.91 \%$, respectively). ${ }^{1}$

Further inspection revealed that FA rates also varied as a function of the two preceding stimuli. In particular, mean FA rates were much higher following two successive stimuli containing complementary target features (i.e., $\mathrm{C}+\mathrm{O}-$ followed by $\mathrm{C}-\mathrm{O}+$, and $\mathrm{C}-\mathrm{O}+$ followed by $\mathrm{C}+\mathrm{O}-$ ) whose conjunction could form a target (mean $6.5 \%$ ) than following distractor pairs with a repetition of the same target feature [e.g., $\mathrm{C}+\mathrm{O}-$ followed by $\mathrm{C}+\mathrm{O}-, 1.7 \%$, $F(1,11)=34.1, p<.001]$. Further analysis of these "illusory conjunctions" was performed to determine whether their probability related to the interval separating the stimuli with complementary features. The incidence of FAs was similar following pairs in which the two distractors were separated by SOAs shorter than the median (171-307 msec) and longer than the median SOA (321-457 msec). Finally, it should be noted that while FAs in conjunction conditions occurred disproportionately following distractor pairs that could produce the incidence of FAs following such pairs was much less frequent than the accurate binding of the target features. FAs occurred following $6.5 \%$ of stimulus pairs that contained complementary target features, whereas target hit rates averaged more than $99 \%$.

Priming effects. Target responses were examined as a function of the features shared by the target and the immediately preceding ( $\mathrm{T}-1)$ stimulus to evaluate priming. No significant priming effects were seen in orientation or color conditions. However, both the color and the orientation of the $\mathrm{T}-1$ stimulus influenced responses in conjunction conditions, as shown in Tables 5A-5B. ${ }^{2}$ Responses were slowed (by $31 \mathrm{msec}$ ) when the T-1 stimulus shared target color $[F(1,11)=19.4, p<.001]$. Responses were also slowed when the T-2 or T-3 stimulus shared target color [respectively, $+10 \mathrm{msec}, F(1,11)=2.75, p<.15$, and $+18 \mathrm{msec}, F(1,11)=5.43, p<.05]$. In contrast, responses were speeded when the T-1 stimulus shared target orientation [ $-28 \mathrm{msec}, F(1,11)=15.1, p<.005]$. Orientation priming dissipated more rapidly than color priming: No significant orientation effects were seen at T-2 or T-3 positions.

\section{Discussion}

Processing of single features. Subjects found it easier to process color than orientation: Responses were $67 \mathrm{msec}$ faster in color than in orientation conditions and error and FA rates were also lower in color conditions. Since the stimuli, responses, and task demands were similar in color and orientation conditions, response differences can be ascribed to differences in feature processing time.

Table 4

Mean Reaction Times (in Milliseconds) and Feature Conjunction Times (FCTs) for Targets of Different Colors and Orientations in Color, Orientation, and Conjunction Conditions

\begin{tabular}{lcccr}
\hline \multicolumn{1}{c}{ Stimulus } & \multicolumn{3}{c}{ Condition } \\
\cline { 2 - 4 } & Color & Orientation & Conjunction & FCT \\
\hline Red/vertical & 359 & 404 & 426 & 22 \\
Red/horizontal & 372 & 419 & 448 & 29 \\
Red/diagonal & 366 & 454 & 458 & 4 \\
Green/vertical & 446 & 474 & 513 & 39 \\
Green/horizontal & 435 & 500 & 537 & 37 \\
Green/diagonal & 431 & 532 & 537 & 5 \\
Yellow/vertical & 430 & 487 & 525 & 38 \\
Yellow/horizontal & 431 & 506 & 487 & -19 \\
Yellow/diagonal & 420 & 514 & 514 & 0 \\
\hline
\end{tabular}


Table 5A

Mean Reaction Times (RTs, in Milliseconds) for Targets of Different Colors in Conjunction Conditions as a Function of the Features Shared With the Stimulus Preceding the Target

\begin{tabular}{lccc}
\hline \multirow{2}{*}{ Target } & \multicolumn{2}{c}{ Preceding Color } & \\
\cline { 2 - 3 } Color & Same & Different & $\Delta$ RT \\
\hline Red & 459 & 414 & +45 \\
Green & 544 & 507 & +37 \\
Yellow & 501 & 492 & +9 \\
\hline
\end{tabular}

Systematic differences were seen in responses to the individual color and orientation features. Red stimuli were processed more rapidly than equiluminant green or yellow stimuli, consistent with previous results (Jones \& Wilkinson, 1975). This is unsurprising: Red was the most saturated color and was subjectively brighter than the other stimuli (Helmholz-Kohlraush effect; see Wyszecki \& Thomas, 1986). Differences in responses for different stimuli may also have reflected chromatic contrast between the stimuli and the background that paralleled their relative RTs. In orientation conditions, vertical stimuli were processed more rapidly than horizontal or diagonal stimuli. This is also consistent with previous reports (Maffei \& Campbell, 1970).

Feature effects provided evidence of hierarchical analysis. During orientation conditions, differences in responses for the different colors persisted: Subjects processed red stimuli faster than green or yellow stimuli. However, during color and conjunction conditions, differences associated with responding to different orientations disappeared. The fact that stimulus color influenced the time required to process orientation may reflect the hierarchical nature of the stimuli themselves: Since the stimuli and background were equiluminant, orientation was defined by color contrast.

Visual feature conjunction. Responses in conjunction conditions were $84 \mathrm{msec}$ slower than responses in color conditions and $17 \mathrm{msec}$ slower than responses in orientation conditions. Since orientation was, on average, the slowest processed single feature, this yielded an FCT estimate of $17 \mathrm{msec}$. As expected, the FCT was lower than the search slopes (20-40 msec/item) typically found in conjunction conditions of visual search tasks. Since attention remained focused on a common, unchanging location in Experiment 1 , estimates of FCT would be expected to be less than slopes in visual search that include the time needed to displace attention from one display element to another.

Table 5B

Mean Reaction Times (RTs, in Milliseconds) for Targets of Different Orientations in Conjunction Conditions as a Function of the Features Shared With the Stimulus Preceding the Target

\begin{tabular}{lccc}
\hline \multirow{2}{*}{$\begin{array}{c}\text { Target } \\
\text { Orientation }\end{array}$} & \multicolumn{2}{c}{ Preceding Orientation } & \\
\cline { 2 - 3 } & Same & Different & $\Delta \mathrm{RT}$ \\
\hline Vertical & 459 & 478 & -19 \\
Diagonal & 458 & 500 & -42 \\
Horizontal & 455 & 478 & -23 \\
\hline
\end{tabular}

However, the small magnitude of mean FCTs calls into question the P/S model. In order to avoid the occurrence of "negative FCTs" on individual trials, the P/S model would require a much larger FCT than that obtained. Moreover, the FCT estimates were likely inflated because target detection in conjunction conditions depended on the outputs of both color and orientation processing, but FCT was always estimated by comparing conjunction and orientation conditions. This would result in an overestimation of FCT on trials where orientation processing terminated before color processing. Finally, target detection in conjunction conditions would have been slowed by increased distractor heterogeneity (Duncan \& Humphreys, 1989). Since there were more distractors in conjunction conditions (eight) than in single-feature conditions (six), distractor heterogeneity could have increased conjunction RTs and hence inflated FCT estimates.

It is possible that slopes in visual search tasks reflect only the time required to displace attention. Once spatially focused on a stimulus, feature conjunction may occur instantaneously and automatically (Treisman \& Gelade, 1980). Since attention remained focused on a single spatial location in our experiment, features might have been conjoined as soon as the processing of the slower feature terminated: that is, the "real" FCT might have been zero. Positive FCT measures would be explained by occasional trials where color processing finished after orientation processing and by increased distractor heterogeneity in conjunction conditions.

However, response variances were inconsistent even with a $\mathrm{P} / \mathrm{S}$ with instantaneous conjunction. According to the $\mathrm{P} / \mathrm{S}$ model, response variance should be reduced in conjunction conditions in comparison with orientation conditions. In fact, variance was increased in conjunction conditions.

The results can be more adequately explained by a parallel/interactive $(\mathrm{P} / \mathrm{I})$ model, in which the processing of different features interacts during at early stages of analysis (see, e.g., Hillyard \& Münte, 1984). The P/I model accounts for short RTs in conjunction conditions by assuming that the processing of orientation was facilitated for stimuli of target color. This makes it possible for subjects to process color and orientation of some stimuli in conjunction conditions faster than orientation alone is processed in orientation conditions. The $\mathrm{P} / \mathrm{I}$ model is consistent with guided search models in that feature processing is interactive (see, e.g., Cohen, 1993; Wolfe, 1994). However, the results of the present experiment suggest that the interaction is not restricted to the spatial displacement in search tasks but that processing interactions may also influence the processing of stimulus features when attention remains spatially focused.

The mechanism of such interaction remains uncertain. One possibility is that it reflects a reduction in processing load in conjunction conditions in comparison with orientation conditions. This might occur if processing of different features was parallel and self-terminating (Hansen \& Hillyard, 1983), so that processing in conjunction con- 
ditions would terminate as soon as evidence accrued along either feature dimension that a distractor was not a target. This would make it possible in conjunction conditions to terminate orientation processing for distractors lacking target color. Thus, in conjunction conditions only one third of the stimuli would require orientation processing, reducing the overall processing load along the more difficult (orientation) dimension.

This explanation is consistent with the priming effects seen in conjunction conditions. Target-colored distractors would be expected to undergo a longer, more sustained analysis. Thus, when a target followed a distractor of target color, the target would often be presented before the processing of the preceding stimulus had finished. In contrast, distractors of target orientation (hence not of target color) could be rejected more rapidly than the average distractor and hence would be associated with positive priming. The priming effects may also reflect the Fletcher and Rabbitt (1978; Krueger \& Shapiro, 1981) bypass rule. That is, subjects' responses are slowed if (1) the stimulus preceding the target and the target share features and (2) the subject is required to withhold responses to the preceding target-like distractor. Finally, negative priming may have reflected a motion illusion. Successive bars of the same color, but with different orientations, give the illusion of a rotating bar. Since subjects may have initially inhibited their response to the incorrectly oriented but correctly colored bar, the response slowing observed may reflect carryover effects of object-based inhibition (Vecera \& Farah, 1994).

Miscombinations of features across stimuli. Given the high rate of stimulus delivery in the present study, it is likely that stimuli were often presented before the processing of the previous stimulus was complete (Duncan, Ward, \& Shapiro, 1994). This may have facilitated a confusion in the assignment of features to stimuli. This is consistent with the observation that FAs in conjunction conditions occurred disproportionately following stimulus pairs that could produce illusory conjunctions. Keele, Cohen, Ivry, Liotti, and Yee (1988) compared the incidence of illusory conjunctions for stimuli presented successively in time at similar locations in space with those for stimuli presented simultaneously at different spatial locations. They found that illusory conjunctions were more common for stimuli presented sequentially at common locations.

\section{EXPERIMENT 2}

Visual search paradigms that have yielded insight into the mechanisms of feature conjunction cannot be transposed to the auditory modality because the simultaneous presentation of multiple auditory signals results in peripheral masking at the cochlea. In Experiment 2 we used the RSP paradigm to study auditory feature conjunction.

RSP paradigms have been widely used in event-related brain potential (ERP) studies of auditory selective attention (Hansen \& Hillyard, 1983; Hillyard, Hink, Schwent, \& Picton, 1973). In these experiments, subjects typically attend to a stream of frequent "standard" stimuli, defined by one or more features, and respond to occasional deviant target stimuli differing slightly along a different feature. For example, they may attend to tones of a particular ear of delivery and frequency in order to detect occasional targets of longer duration (see, e.g., Woods, Alho, \& Algazi, 1994).

ERP experiments have provided evidence supporting P/I models: ERP signs of feature conjunction begin at short latencies, shortly after the ERP signs of individual feature processing begin, and continue in parallel with ERP signs of individual feature processing for hundreds of milliseconds (Woods et al., 1994). Miscombination of features between successive stimuli is also predicted by the ERP data. Electrophysiological signs of the processing of distractors with one target feature may persist for $500 \mathrm{msec}$ or more. Thus, if stimuli are presented at shorter interstimulus intervals (ISIs), the ERP data imply that successive stimuli are undergoing concurrent simultaneous analysis (Woods \& Alain, 1993).

Unfortunately, the RT data from previous RSP/ERP experiments provide little direct insight into the time course of processing of target features because RTs to targets have largely reflected the difficulty in discriminating deviant and standard tones. In the present experiment, we attempted to derive estimates of the time course of processing of frequency and location features of the auditory signals using behavioral procedures similar to those used in Experiment 1, Thus, in single-feature conditions, subjects responded to targets defined by tone frequency or location (ear of delivery), whereas in conjunction conditions they responded to targets defined by the combination of frequency and location features.

\section{Method}

Subjects. Twelve subjects ( 6 males and 6 females, age range 21-45 years) participated, including 4 who had participated in Experiment 1 . All were right-handed with normal hearing (thresholds less than $15 \mathrm{~dB}$ normal hearing level [nHL] from 250 to $4000 \mathrm{~Hz}$ ).

Stimuli and task. Figure 2 shows a sample of the stimulus sequence. Nine different loudness-balanced tone bursts were used (50msec duration with 5-msec rise/fall times). These were derived from the combination of three tone frequencies $(250,612$, or $1500 \mathrm{~Hz})$ and three locations (left ear, binaural, or right ear). The tones were equated psychophysically in loudness: Monaural tone bursts were presented at $87 \mathrm{~dB}$ SPL for the 250 - and $612-\mathrm{Hz}$ tones, and at $85 \mathrm{~dB}$ SPL for the $1500-\mathrm{Hz}$ tone. Binaural tones were attenuated by $6 \mathrm{~dB}$ in each ear in comparison with monaural tones. SOAs, target probabilities, and mean intertarget intervals were identical to those used in Experiment 1. Subjects responded to a prespecified tone feature (either frequency or location) or to a prespecified conjunction of features (e.g., 1500-Hz tone, left ear).

There was a total of 15 different conditions: three frequency conditions, three location conditions, and nine conjunction conditions. Stimuli were presented in blocks containing 984 stimuli and lasting $4.43 \mathrm{~min}$. Each frequency and location condition was repeated for three blocks, and each conjunction condition was run for one block, so that a total of 27 blocks $(26,568$ stimuli including 2,952 targets) were presented in all. The order of presentation of blocks was counterbalanced across subjects. In all conditions, subjects were encouraged to be both fast and accurate in their responses.

Data analysis. Data analysis was similar to that in Experiment 1. However, because auditory RTs tended to be faster than visual RTs, hits were defined as buttonpresses occurring $200-800 \mathrm{msec}$ after 


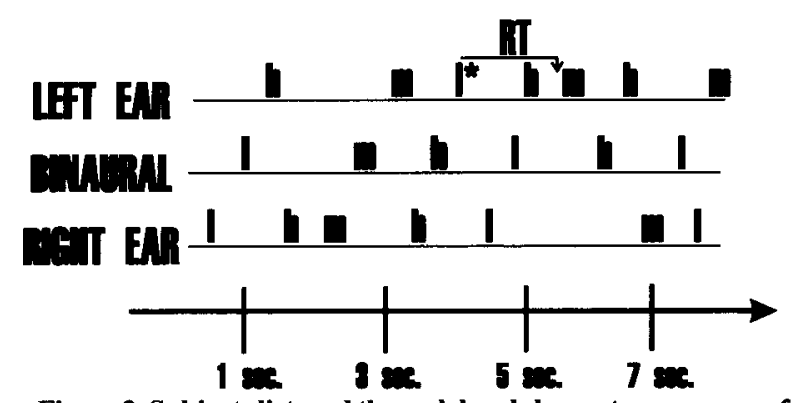

Figure 2. Subjects listened through headphones to sequences of loudness-matched tones of three frequencies presented randomly to the left ear, right ear, or center of the head. In frequency conditions, subjects responded to all tones of a designated frequency. In location conditions, they responded to tones of a designated location. In conjunction conditions, they responded only to tones of a prespecified frequency and location. A segment of the conjunction sequence, “250 Hz left ear," is shown. 1, $250 \mathrm{~Hz}$; m, $612 \mathrm{~Hz}$; h, 1500 Hz. RT, reaction time.

targets (rather than 200-1,000 msec). FAs were defined as responses occurring without a target during the preceding $800 \mathrm{msec}$.

\section{Results}

Overview. Mean RTs across all conditions averaged $413 \mathrm{msec}$ (range across subjects, $326-540 \mathrm{msec}$ ). Subjects were highly accurate, missing only $2.3 \%$ of the targets. FAs occurred on $1.0 \%$ of all nontarget presentations.

Performance measures for frequency, location, and conjunction conditions are presented in Table 6 . There were large differences in RT among conditions: RTs were fastest in frequency conditions $(340 \mathrm{msec})$, intermediate in conjunction conditions ( $425 \mathrm{msec})$, and slowest in location conditions $(475 \mathrm{msec})[F(2,22)=34.9, p<.001]$. Specific comparisons between conditions showed that responses were significantly faster in frequency than in conjunction conditions $[F(1,11)=21.0, p<.001]$. This effect was consistently observed in each of the 12 subjects (range $=18-206 \mathrm{msec}$ ). Responses were also significantly faster in conjunction than in location conditions $[F(1,11)=$ $54.3, p<.001]$. This effect was also observed in each subject $($ range $=27-136 \mathrm{msec})$.

Speed-accuracy tradeoffs did not account for the slowing of responses in location conditions: Error rates in location conditions were slightly higher than those in frequency or conjunction conditions, but none of the differences reached significance. However, a significant condition effect was found for FAs [ frequency $=0.5 \%$, conjunction $=1.3 \%$, location $=1.3 \%, F(2,22)=13.3, p<.003]$. Pairwise comparisons showed that the only significant differences were between frequency and the two other conditions $[F(1,11)=$ 16.5 and $20.1, p<.01$ for both comparisons].

Differences in the processing of individual tone features. Table 7 shows the performance measures in different conditions as a function of tone frequency. There was no overall main effect of tone frequency on RT. However, there was a significant condition $\times$ frequency interaction $[F(4,44)=5.78, p<.001]$, due primarily to the fact that the $1500-\mathrm{Hz}$ tone had relatively faster responses than other frequencies in conjunction $(-25 \mathrm{msec})$ and frequency conditions $(-12 \mathrm{msec})$, but in location conditions, responses to $612-\mathrm{Hz}$ tones were speeded $(-33 \mathrm{msec}$; see Table 7).

Table 8 shows performance measures in the different conditions as a function of tone location. Responses were slowed following binaural targets (mean RT $=\mathbf{4 4 6} \mathrm{msec}$ ) in comparison with left ( $400 \mathrm{msec}$ ) or right ( $394 \mathrm{msec}$ ) ear targets, producing a significant location effect $[F(2,22)=33.0$, $p<.001]$. There was also a highly significant condition $\times$ location interaction $[F(4,44)=47.2, p<.001]$. As shown in Table 8, subjects were faster in responding to binaural than to monaural targets in frequency conditions $[-12 \mathrm{msec}$, $F(1,11)=10.5, p<.008]$, but were slower in responding to binaural targets in conjunction conditions $[+29 \mathrm{msec}$, $F(1,11)=4.7, p=.052]$ and particularly in location conditions $[+134 \mathrm{msec}, F(1,11)=92.1, p<.001]$. Responses to binaural targets were delayed in location in comparison with conjunction conditions $[F(1,11)=139.2, p<.0001]$, whereas the responses to monaural targets showed only a tendency toward delay $[F(1,11)=2.8, p<.12]$.

Feature conjunction time. An estimate of FCT was derived for each target stimulus by subtracting the mean RTs to stimuli in the slowest single-feature condition (location) from the mean RTs to the same stimulus in the conjunction condition. FCTs for different target stimuli are presented in Table 9. Responses were faster in conjunction than in location conditions, producing a mean FCT estimate of $-50 \mathrm{msec}$. FCT measures differed for different frequencies $[F(2,22)=9.4, p<.001]$; FCTs were less negative for $612-\mathrm{Hz}$ tones $(-21 \mathrm{msec})$ than for 250 $(-53 \mathrm{msec})$ or $1500-\mathrm{Hz}(-75 \mathrm{msec})$ tones. FCTs also varied with location $[F(2,22)=36.7, p<.001]$, being more negative following binaural $(-123 \mathrm{msec})$ than following monaural $(-14 \mathrm{msec})$ targets.

False alarms. In frequency conditions, the FA rate was low (mean $0.5 \%$ ), and FAs occurred with similar probabilities following different nontarget frequencies. In location conditions, FAs were more frequent $(1.3 \%)$ and occurred more often following binaural than following left or right monaural tones $[F(1,11)=6.2, p<.03]$.

Illusory conjunctions. In conjunction conditions, FAs tended to be more common following distractors with at least one target feature (FA rate $2.0 \%$ ) than following distractors with no target features (1.2\%). As in Experiment 1 , FAs were more likely to occur following distractor pairs containing complementary target features (FA rate $2.7 \%$ ) than following pairs with a repetition of the same target feature [FA rate $1.7 \%, F(1,11)=11.3, p<.01$ ]

Table 6

Mean Percentage of Misses and False Alarms and Reaction Times (RTs) for the Different Conditions of Experiment 2, Averaged Over Stimuli of Different Frequencies and Locations

\begin{tabular}{lccc}
\hline Condition & Misses (\%) & False Alarms (\%) & Mean RT (msec) \\
\hline Frequency & 2.7 & 0.5 & 340 \\
Location & 3.1 & 1.3 & 475 \\
Conjunction & 0.9 & 1.3 & 425 \\
\hline
\end{tabular}


Table 7

Mean Reaction Times (in Milliseconds) for

Targets of Different Frequencies in Frequency, Location, and Conjunction Conditions

\begin{tabular}{cccc}
\hline Target & \multicolumn{3}{c}{ Condition } \\
\cline { 2 - 4 } Frequency & Frequency & Location & Conjunction \\
\hline $250 \mathrm{~Hz}$ & 342 & 487 & 434 \\
$612 \mathrm{~Hz}$ & 346 & 454 & 432 \\
$1500 \mathrm{~Hz}$ & 332 & 485 & 409 \\
\hline
\end{tabular}

Priming effects. Mean RTs varied significantly as a function of the tone preceding the target. For example, responses were slowed in location conditions when the tone preceding the target shared target frequency. This slowing was seen for left ear tones [mean effect $+48 \mathrm{msec}, F(1,10)=$ $58.0, p<.001]$, binaural tones [ $+28 \mathrm{msec}, F(1,10)=6.7$, $p<.05]$, and right ear tones $[+52 \mathrm{msec}, F(1,10)=33.4$, $p<.001]$. Responses were also delayed in conjunction conditions (see Tables 10A-10B) when targets followed tones sharing target frequency [+43 msec, $F(1,10)=54.8, p<$ $.001]$. A delay was also evident following distractors that occurred two or three positions before the target [T-2, $+31 \mathrm{msec}, F(1,10)=31.2, p<.001 ; \mathrm{T}-3,+20 \mathrm{msec}$, $F(1,10)=16.3, p<.01]$. The relative locations of the tone preceding the target and the target did not significantly influence RT in any condition.

\section{Discussion}

Processing of single auditory features. Responses were faster in frequency than in location conditions. Indeed, the slowest frequency condition was faster than the fastest location condition, and slowing in location relative to frequency conditions was seen for all subjects. This difference did not reflect speed-accuracy tradeoffs: Subjects missed fewer targets and made fewer FAs in frequency than location conditions. These results are in apparent contradiction to previous studies that have emphasized the importance of location cues in guiding auditory selective attention. For example, Näätänen, Porkka, Merisalo, and Ahtola (1980) found that subjects were faster to respond to left or right ear tones than they were to respond to large differences (up to five octaves) in tone frequency when tones were presented at long $(5 \mathrm{sec})$ ISIs. However, when high rates of auditory signal delivery are used, frequency is often a more effective cue than location in both attracting attention and maintaining an attentional focus (Woods, Alain, Diaz, Ogawa, \& Rhodes, 1997).

Subjects were faster to process high- than low-frequency tones in frequency and conjunction conditions. This is consistent with previous electrophysiological results suggesting that high-frequency tones are processed more rapidly at all stages of the nervous system and generate faster responses as a result (Woods, Alain, Covarrubias, \& Zaidel, 1993). This may reflect the existence of a subset of large, rapidly conducting auditory neurons that are tuned to high-frequency sounds.

Performance differences were also found for the different tone locations: Responses in binaural conditions were slowed in comparison with monaural conditions. This is not surprising: Binaural tones were intermediate in location between two monaural distractors and shared cochlear excitation patterns with each. In location conditions, subjects were also slow to respond to $1500-\mathrm{Hz}$ tones. This probably reflects well-known difficulties in localizing sounds in the $1500-2500 \mathrm{~Hz}$ frequency range (Scharf \& Houtsma, 1986).

Auditory feature conjunction. Subjects were faster to detect targets in frequency conditions than in conjunction or location conditions, but were faster to detect targets in conjunction than in location conditions. This RT difference produced negative mean FCTs.

These results are incompatible with $\mathrm{P} / \mathrm{S}$ models. $\mathrm{P} / \mathrm{S}$ models require that RTs in conjunction conditions equal or exceed RTs in the slowest single-feature condition. The fact that subjects were faster to process location and frequency in conjunction conditions than location alone in location conditions implies that the processing of single features occurred interactively. In Experiment 2, restricting attention to a single-tone frequency apparently facilitated the analysis of tone location. This was particularly true in binaural conditions: RTs were 123 msec faster in conjunction than in location conditions. As in Experiment 1, observed response differences probably underestimated differences in processing time in conjunction and location conditions since (1) FA rates were higher in location conditions, (2) some overlap was evident in RT distributions of frequency and location conditions, and (3) distractor complexity was greater in conjunction than in location conditions.

The exact mechanism whereby location processing was improved for tones of target frequency remains obscure. Perhaps subjects were able to suppress processing of tones of nontarget frequencies by inhibiting frequency-irrelevant regions of auditory cortex (Alho, Woods, \& Algazi, 1994; Woods, 1990), or perhaps the processing of tones of target frequency was enhanced (Alain \& Woods, 1994). In any case, the behavioral results are consistent with parallel self-terminating models (Hansen \& Hillyard, 1983), a subclass of $\mathrm{P} / \mathrm{I}$ models. If we assume that location analysis was a difficult, capacity-limited process, the high rate of stimulation might reveal capacity limitations when successive stimuli required detailed location analysis. In conjunction conditions, outputs from the faster frequencyanalysis stage might permit subjects to terminate processing before location analysis has finished, reducing overall processing load and speeding responses. Alternatively, the results may be explained by a parallel, exhaustive model

Table 8

Mean Reaction Times (in Milliseconds) for

Targets of Different Locations in Frequency, Location, and Conjunction Conditions

\begin{tabular}{lccc}
\hline \multirow{2}{*}{$\begin{array}{c}\text { Target } \\
\text { Location }\end{array}$} & Frequency & Location & Conjunction \\
\cline { 2 - 3 } & 344 & 434 & 423 \\
Left & 332 & 565 & 442 \\
Binaural & 343 & 427 & 410 \\
Right & & & \\
\hline
\end{tabular}


Table 9

Mean Reaction Times (in Milliseconds) and Feature Conjunction Times (FCTs) for Targets of Different Frequencies and Locations in Frequency, Location, and Conjunction Conditions

\begin{tabular}{ccccr}
\hline & \multicolumn{3}{c}{ Condition } & \\
\cline { 2 - 4 } Stimulus & Frequency & Location & Conjunction & FCT \\
\hline $250 \mathrm{~Hz} /$ Left & 355 & 476 & 432 & -44 \\
$612 \mathrm{~Hz} /$ Left & 344 & 411 & 426 & 15 \\
$1500 \mathrm{~Hz} /$ Left & 331 & 416 & 412 & -4 \\
$250 \mathrm{~Hz} /$ Binaural & 325 & 551 & 446 & -105 \\
$612 \mathrm{~Hz} /$ Binaural & 342 & 540 & 448 & -92 \\
$1500 \mathrm{~Hz} /$ Binaural & 329 & 602 & 433 & -169 \\
$250 \mathrm{~Hz} /$ Right & 346 & 434 & 425 & -9 \\
$612 \mathrm{~Hz} /$ Right & 352 & 410 & 423 & 13 \\
$1500 \mathrm{~Hz} /$ Right & 336 & 437 & 383 & -54 \\
\hline
\end{tabular}

in which the processing of target frequency and location features is mutually facilitatory.

Since both $\mathrm{P} / \mathrm{I}$ models imply cross-modulation of feature processing, they predict that conjunction-specific processing operations should begin at short latencies. Evidence from ERPs is consistent with this suggestion: ERP conjunction effects begin 40-60 msec after ERP signs of single-feature processing, consistent with suggestions that conjunction effects may influence the processing of individual stimulus features (Woods et al., 1994).

Priming effects. Negative priming for target frequency was observed in the present experiment. This is consistent with the hypothesis that the processing of stimuli of nontarget frequencies was gated before full analysis. Perhaps only those distractors of target frequency underwent more detailed location processing and captured attention (Jonides \& Yantis, 1988). Thus, attention might have been redirected to location only following tones of target frequency. The negative priming would thus reflect the time required for the spatial redeployment of attention to the target location. Alternatively, the results may reflect object-based inhibition similar to that suggested in Experiment 1: Successive tones of target frequency occurring first at nontarget and then at target locations gave the illusion of a moving tone. The fact that negative priming occurred when the targetfrequency tone occurred two or three positions before the target suggests that object inhibition and/or spatial reorientation effects persisted for almost $1.0 \mathrm{sec}$.

Illusory conjunctions. As in Experiment 1, FAs in conjunction conditions occurred disproportionately following stimulus pairs that contained complementary target features. FAs remained rare, even in this case: FAs occurred following less than $3 \%$ of stimulus pairs that contained complementary target features, whereas target hit rates averaged over $98 \%$. ERP studies suggest that the processing of individual tone features persists for $350-450 \mathrm{msec}$ (Woods et al., 1994). Given the short intervals between successive stimuli in the present experiment (mean SOA = $300 \mathrm{msec}$ ), features from successive tones would undergo a variable period of simultaneous processing, which would appear to produce vulnerability to feature miscombination.

\section{GENERAL DISCUSSION}

The pattern of results was similar in both visual and auditory modalities. One stimulus dimension, color in Experiment 1 and frequency in Experiment 2, was easy to process and produced correspondingly rapid responses in single-feature conditions. The other dimension, orientation in Experiment 1 and location in Experiment 2, was more difficult and produced slower responses and higher FA rates.

The similarities between color and frequency extend further. Negative priming was seen for both attributes. This can be explained by a parallel self-terminating attention model (Hansen \& Hillyard, 1983) in which the processing of a stimulus terminates as soon as evidence accumulates that it is not a target. In the visual modality, this decision would be made first for stimulus color, in the auditory modality, it would be made first for stimulus frequency. Specifically, the processing of stimuli not sharing target color in Experiment 1 or target frequency in Experiment 2 would be attenuated at a relatively early stage. Conversely, longer, more sustained processing would presumably be required of stimuli sharing target color or frequency. ERP recordings demonstrate that distractors sharing target color (Woods, Thomas, Han, Yund, \& Ogawa, 1997) undergo many of the processing stages characteristic of targets, up to latencies of more than $350 \mathrm{msec}$. In contrast, ERP attention effects are attenuated for distractors sharing target orientation (but not target color). Given the short mean SOAs in the present experiments, when a target was preceded by distractor of target color or target frequency, it would often be delivered before the processing of the distractor had terminated. This would explain the delay associated with target processing when the target followed distractors of target color or frequency.

The results from both modalities are incompatible with the $\mathrm{P} / \mathrm{S}$ model. In Experiment 1, responses in conjunction conditions were faster than responses in corresponding orientation conditions for certain targets and certain subjects. In Experiment 2, responses were systematically faster in conjunction than in location conditions. Correcting for speed-accuracy tradeoffs, distractor complexity effects, and overlap in RT distributions in single-feature conditions would have made FCTs smaller or negative in Experiment 1 and would have produced even more markedly negative FCTs in Experiment 2.

The fact that responses in conjunction conditions were often faster than responses in single-feature conditions

Table 10A

Mean Reaction Times (RTs, in Milliseconds) for Targets of Different Frequencies in Conjunction Conditions as a Function of the Features Shared With the Stimulus Preceding the Target

\begin{tabular}{cccc}
\hline \multirow{2}{*}{$\begin{array}{c}\text { Target } \\
\text { Frequency }\end{array}$} & \multicolumn{2}{c}{ Preceding Frequency } & \\
\cline { 2 - 3 } & Same & Different & $\Delta$ RT \\
\hline $250 \mathrm{~Hz}$ & 459 & 422 & +37 \\
$612 \mathrm{~Hz}$ & 465 & 428 & +37 \\
$1500 \mathrm{~Hz}$ & 461 & 403 & +58 \\
\hline
\end{tabular}


Table 10B

Mean Reaction Times (RTs, in Milliseconds) for Targets of Different Locations in Conjunction Conditions as a Function of the Features Shared With the Stimulus Preceding the Target

\begin{tabular}{lccr}
\hline \multirow{2}{*}{$\begin{array}{c}\text { Target } \\
\text { Frequency }\end{array}$} & \multicolumn{2}{c}{ Preceding Frequency } & \\
\cline { 2 - 3 } & Same & Different & SRT \\
\hline Left & 433 & 431 & +2 \\
Binaural & 441 & 438 & +3 \\
Right & 414 & 424 & -10 \\
\hline
\end{tabular}

(particularly in the auditory modality) suggests that the processing of the individual features interacted. When attention was focused along a highly discriminable dimension (e.g., frequency), processing improved for other features (e.g., location) of the same stimulus.

Previous experiments using physiological measures have shown that stimulus selection in filtering tasks depends on the modulation of cortical regions that process the attended features. For example, attending to stimulus color modulates processing in color-responsive cortical regions (Corbetta, Miezin, Dobmeyer, Shulman, \& Petersen, 1991). Electrophysiological recordings show that these changes are evident at short latencies and therefore must reflect tonic inhibitory/excitatory modulation of feature processing fields (Woods, 1990).

When subjects attend to a target defined by the conjunction of two highly discriminable features, auditory ERPs show short-latency enhancements of processing along both feature dimensions, with conjunction-specific ERP effects seen at short latencies (e.g., $120 \mathrm{msec}$ ) that are compatible with enhanced processing along both feature dimensions (Woods et al., 1994).

There are several possible explanations for the mutually facilitatory interaction between feature fields. For example, parallel self-terminating models (Hansen \& Hillyard, 1983) could explain the results as a consequence of an economization of processing resources. Evidence from the more salient dimensions that a stimulus was not a target would allow subjects to abort processing, reducing processing load in conjunction conditions relative to the more difficult single-feature conditions. Alternatively, projections from one feature processing field might facilitate processing along other feature fields. In that case, reafferent projections (Finkel \& Edelman, 1989) from one cortical field to another (possibly relayed through a central field, or master map, Treisman, 1988) might facilitate processing of the less discriminable feature dimensions. ERP evidence from auditory tasks is consistent with this reafferent model: Processing along the less salient feature location dimension is not terminated abruptly when the distractor can be discriminated from the target along the more salient frequency dimension; rather, it continues for at least $400 \mathrm{msec}$ (Woods et al., 1994). The reafferent, topdown excitation back onto feature maps would be particularly effective in facilitating the processing of less salient features, since, in comparison with weak bottom-up inputs, reafferent inputs might arrive earlier and have relatively greater weighting. Hence, these effects would be- come evident in conditions where one feature was easy to process and the other difficult, thus making it sometimes faster to process two stimulus features than to process one.

\section{REFERENCES}

AlaIN, C., \& Woons, D. L. (1994). Signal clustering modulates auditory cortical activity in humans. Perception \& Psychophysics, 56, 501-516.

Alho, K., Woods, D. L., \& Algazi, A. (1994). Processing of auditory stimuli during auditory and visual attention as revealed by eventrelated potentials. Psychophysiology, 31, 469-479.

CoHEN, A. (1993). Asymmetries in visual search for conjunctive targets. Journal of Experimental Psychology: Human Perception \& Performance, 19, 775-797.

Corbetta, M., Miezin, F. M., Dobmeyer, S., Shulman, G. L., \& PeTERSEN, S. E. (1991). Selective and divided attention during visual discriminations of shape, color, and speed: Functional anatomy by positron emission tomography. Journal of Neuroscience, 11, 2383-2402.

Driver, J., Baylis, G. C., \& Rafal, R. D. (1992). Preserved figureground segregation and symmetry perception in visual neglect. Nature, 360, 73-75.

DunCAN, J., \& Humphreys, G. W. (1989). Visual search and stimulus similarity. Psychological Review, 96, 433-458.

Duncan, J., Ward, R., \& Shapiro, K. (1994). Direct measurement of attentional dwell time in human vision. Nature, 369, 313-315.

FinKel, L. H., \& Edelman, G. M. (1989). Integration of distributed cortical systems by reentry: A computer simulation of interactive functionally segregated visual areas. Journal of Neuroscience, 9, 3188-3208.

FLETCHER, B., \& RABBITT, P. M. A. (1978). The changing pattern of perceptual analytic strategies and response selection with practice in a two-choice reaction time task. Quarterly Journal of Experimental Psychology, 30, 417-427.

HANSEN, J. C., \& Hillyard, S. A. (1983). Selective attention to multidimensional auditory stimuli. Journal of Experimental Psychology: Human Perception \& Performance, 9, 1-19.

Hillyard, S. A., Hink, R. F., Schwent, V. L., \& Picton, T. W. (1973). Electrical signs of selective attention in the human brain. Science, 182 , 177-179.

HillyaRd, S. A., \& MünTE, T. F. (1984). Selective attention to color and location: An analysis with event-related brain potentials. Perception \& Psychophysics, 36, 185-198.

Jones, P. D., \& Wilkinson, H. (1975). Latency differences to monochromatic stimuli measured by disjunctive reaction time. Perceptual \& Motor Skills, 41, 55-59.

JoNIDES, J., \& YANTIS, S. (1988). Uniqueness of abrupt visual onset in capturing attention. Perception \& Psychophysics, 43, 346-354.

KeEle, S. W., Cohen, A., Ivry, R., LiotTi, M., \& Yee, P. (1988). Tests of a temporal theory of attentional binding. Journal of Experimental Psychology: Human Perception \& Performance, 14, 444-452.

KLEIN, R., \& FARRELL, M. (1989). Search performance without eye movements. Perception \& Psychophysics, 46, 476-482.

KRUEGER, L. E., \& SHAPIRo, R. G. (1981). Intertrial effects of samedifferent judgements. Quarterly Journal of Experimental Psychology, 33A, 241-265.

MAFFEI, L., \& CAMPBell, F. W. (1970). Neurophysiological localization of the vertical and horizontal visual coordinates in man. Science, 167, 386-387.

NäÄtÄnen, R., Porkka, R., Merisalo, A., \& Ahtola, S. (1980). Location vs. frequency of pure tones as a basis of fast discrimination. Acta Psychologica, 44, 31-40.

Posner, M. I., \& Driver, J. (1992). The neurobiology of selective attention. Current Opinion in Neurobiology, 2, 165-169.

Prinzmetal, W., Presti, D. E., \& Posner, M. I. (1986). Does attention affect visual feature integration? Journal of Experimental Psychology: Human Perception \& Performance, 12, 361-369.

SCHARF, B., \& HoutsMa, A. J. M. (1986). Audition II. Loudness, pitch, localization, aural distortion, pathology. In K. R. Boff, L. Kaufman, \& J. P. Thomas (Eds.), Handbook of perception and human performance: Vol. I. Sensory processes and perception (pp. 15-1 to 15-60). New York: Wiley. 
Treisman, A. M. (1988). Features and objects: The Fourteenth Bartlett Memorial Lecture. Quarterly Journal of Experimental Psychology, 40, 201- 237.

Treisman, A. M., \& Gelade, G. (1980). A feature-integration theory of attention. Cognitive Psychology, 12, 97-136.

Treisman, A. M., \& Sato, S. (1990). Conjunction search revisited. Journal of Experimental Psychology: Human Perception \& Performance, 16, 459-478.

VeCERA, S. P., \& FARAH, M. J. (1994). Does visual attention select objects or locations? Journal of Experimental Psychology: General, 123, 146-160.

WOLFE, J. M. (1994). Guided Search 2.0: A revised model of visual search. Psychonomic Bulletin \& Review, 1, 202-238.

Woods, D. L. (1990). The physiological basis of selective attention: Implications of event-related potential studies. In J. W. Rohrbaugh, R. Johnson, \& R. Parasuraman (Eds.), Event-related brain potentials: Issues and interdisciplinary vantages (pp. 178-209). New York: Oxford University Press.

Woods, D. L., \& AlAIN, C. (1993). Feature processing during high-rate auditory selective attention. Perception \& Psychophysics, 53, 391-402.

Woods, D. L., Alain, C., Covarrubias, D., \& Zaidel, O. (1993). Frequency-related differences in the speed of human auditory processing. Hearing Research, 66, 46-52.

Woods, D. L., Alain, C., Diaz, R., Ogawa, K. H., \& Rhodes, D. (1997). Factors influencing frequency and location guidance of auditory selective attention. Manuscript submitted for publication.

Woods, D. L., AlHo, K., \& AlgaZI, A. (1994). Stages of auditory feature conjunction: An event-related brain potential study. Journal of Experimental Psychology: Human Perception \& Performance, 22, 81-94.

Woods, D. L., Thomas, S. J., Han, S., Yund, E. W., \& Ogawa, K. H.
(1997). Feature processing and conjunction in a non-spatial visual attention task. Manuscript in preparation.

WyszecKI, G., \& ThomAs, J. P. (1986). Color appearance. In K. R. Boff,

L. Kaufman, \& J. P. Thomas (Eds.), Handbook of perception and human performance: Vol. I. Sensory processes and perception (pp. 9-1 to 9-57). New York: Wiley.

\section{NOTES}

1. Several stimuli could sometimes occur within the 500-msec window preceding the FA. This caused an inflation in the overall rate of FAs in the FA pattern analysis, but did not induce bias in the relative FA rates associated with different distractor types.

2. These effects were operationally confounded since nontargets that shared target color could not share target orientation and vice versa. Since there were eight possible distractors (two sharing orientation, two sharing color, and four sharing neither attribute), if distractors of target color produced an increase in target RT, a decrease one third as large would have been expected following distractors of target orientationeven in the absence of any real orientation effect. For example, if the overall mean RT following all distractors were $460 \mathrm{msec}$, and the mean RT following the distractors of target color were $490 \mathrm{msec}$, then the mean RT following the six distractors lacking target color (including those of target orientation) would be $450 \mathrm{msec}$. However, since the magnitudes of RT change were similar following distractors of target color and orientation, this confounding does not account for the results.

(Manuscript received December 13, 1993; revision accepted for publication February 7, 1997.) 